\title{
TRICHOSPILUS DIATRAEAE CHERIAN \& MARGABANDHU, 1942 (HYMENOPTERA: EULOPHIDAE), UN NUEVO PARASITOIDE DE HYPSIPYLA GRANDELLA (ZELLER, 1848) (LEPIDOPTERA: PYRALIDAE)
}

\author{
TRICHOSPILUS DIATRAEAE CHERIAN \& MARGABANDHU, 1942 \\ (HYMENOPTERA: EULOPHIDAE), A NEW PARASITOID OF HYPSIPYLA \\ GRANDELLA (ZELLER, 1848) (LEPIDOPTERA: PYRALIDAE)
}

\author{
Bruno Zaché1*; Carlos F. Wilcken ${ }^{1}$; Ronelza R. C. Zaché1; \\ Everton P. Soliman ${ }^{1}$; Lorena San Román ${ }^{1}$
}

\begin{abstract}
RESUMEN
Primer reporte de Trichospilus diatraeae (Hymenoptera: Eulophidae) parasitando pupas de lagarta Hypsipyla grandella, principal plaga forestal en América Latina y el Caribe, abriendo nuevas perspectivas para la utilización de este parasitoide en programas de control biológico de lagartas de importancia forestal.
\end{abstract}

Palabras clave: Control biológico; Parasitoide de pupas; Plagas Forestales.

\section{ABSTRACT}

This is the first report of Trichospilus diatraeae (Hymenoptera: Eulophidae) found in the field parasitizing pupae of the Hypsipyla grandella, major forest pest in Latin America and the Caribbean, this offers new perspectives for the use of this parasitoid in biological control programs against caterpillars of forestry importance.

Key words: Biological control; Pupal parasitoid; Forest Pests.

Trichospilus Ferriere, 1930 es un pequeño género en Eulophidae de la tribu Eulophini, con ocho especies descritas (Ferriere 1930; Cherian e Margabandhu, 1942; Boucek, 1976; Zhu et al., 2002). De las ocho especies de este género, tres (Trichospilus boops Boucek, 1976; Trichospilus ferrierei Boucek, 1976; Trichospilus vorax Boucek, 1976) fueron encontradas en África y Trichospilus lutelineatus solamente en Zhejiang en China (Noyes, 2003). Trichospilus striatus Ubaidillah, 2006 y Trichospilus politus Ubaidillah, 2006 fueron descritas para Java y Sulawesi, Indonesia (Ubaidillah, 2006). Trichospilus pupivorus Ferriere, 1930 y Trichospilus diatraeae Cherian e Margabandhu, 1942 ocurren en áreas tropicales y subtropicales (Noyes, 2003) $\mathrm{y}$ han sido estudiadas como agentes potenciales en control biológico de plagas de caña-de azúcar, maíz y algodón en diversos países de África, Asia y América (Boucek, 1976).

T. diatraeae es un parasitoide de pupas gregario, de preferencia de insectos del Orden Lepidoptera (Boucek, 1976). Su presencia ya fue constatada en las familias: Crambidae (Cherian e Margabanghu, 1942), Noctuidae (Etienne e Viette, 1973), Pyralidae (Etienne e Viette, 1973; Boucek, 1976; Bennett et al., 1987), Nymphalidae (Boucek, 1976), Geometridae (Bennett et al., 1987), Pieridae (Torres-Bauza, 1994), Arctiidae (Paron e Berti-Filho, 2000) y Oecophoridae (Oliveira et al., 2001).

T. diatraeae es considerado un parasitoide polífago, siendo principalmente primario. El primer registro de la ocurrencia de T. diatraeae fue en 1942

\footnotetext{
1 Universidade Estadual Paulista Julio Mesquita Filho, Faculdade de Ciências Agronômicas, Departamento de proteção de plantas, CP 18603-970, Botucatu, SP, Brasil. E-mail: bzache@bol.com.br

* Autor para correspondência.
} 
en el hospedero Diatraea venosata, Taladro de tallo en gramíneas. Entre 1963 y 1965 fue introducido en las Islas Maurício y Reunião (Océano Índico), donde fue registrado parasitando Chilo sacchariphagus sacchariphagus Bojer y Sesamia calamistis Hampson (Pyralidae). En la India y en Miamar (Asia) fue registrado parasitando Hypsipyla robusta Moore, Sesamia inferens Walker, Sylepta derogata Fabricius, Pyrausta machaeralis Walker, Scirpophaga incertulas Walker y Chilo sacchariphagus indicus Kapur (Pyralidae) (Boucek, 1976).

En 1963, T. diatraeae fue introducido de la India a Trinidad y Tobago (Antillas) y los EE.UU., utilizando como hospederos Diatraea spp. (Pyralidae) (Bennett et al., 1987). Bordat et al. (1977) realizaron liberaciones de T. diatraeae para el control de plagas de gramíneas en Senegal (África), utilizando el hospedero Chilo suppressalis Walker (Pyralidae). Etienne \& Viette (1973) y Etienne (1975) reportaron T. diatraeae, en las Islas Reunião y Maurício, parasitando las plagas de caña de azúcar Melanitis leda L. y Hedylepta indicata F. (Pyralidae) y Polydesma umbricola Boisduval (Noctuidae) plaga de la conífera Trichopolydesma collutrix. Bournier (1975) utilizó este parasitoide en el control biológico de noctuídeos plagas de algodón en Madagascar (África), empleando como hospedero Anagasta kuehniella Zell. En Comores (África), Breniére et al. (1985) criaron y liberaron T. diatraeae, con el objetivo de controlar Chilo partellus Swinhoe (Pyralidae), plaga de maíz. Rodriguez-del-Bosque \& Smith Junior (1989) realizaron test, con T. diatraeae, en EE.UU. para controlar el Taladro del maíz Diatraea lineolata Walker (Pyralidae). Betbeder-Matibet (1990) estudió la cría masiva Chilo sacchariphagus para la obtención de T. diatraeae. Torres-Bauza (1994) registró al parasitoide atacando pupas de Dismorphia spio (Pieridae) en Ingá vera (Mimosoideae) en Puerto Rico (Antillas).

En Brasil se cree que la introducción fue accidental, registrándose la ocurrencia de T. diatraeae en 1996, en pupas de Arctiidae (Lepidoptera) en Piracicaba, São Paulo (Paron E Berti Filho, 2000). En 2001, este parasitoide fue reportado en pupas de Cerconota anonella (Sepp., 1830) (Lepidoptera: Oecophoridae) en plantaciones de Graviola (Annona muricata L.), en Planaltina, Distrito Federal, Brasil (Oliveira et al., 2001). Recientemente, T. diatraeae fue colectado en pupas de Thyrinteina arnobia (Stoll, 1782) (Lepidoptera: Geometridae), en plantaciones de eucalipto, Minas Gerais (Pereira et al., 2008). En 2010 Zaché et al. constataron a través de pruebas el parasitismo de $T$. diatreae en pupas de Melonolofia consumilaria que es una plaga de gran importancia forestal. Los Prasitoides utilizados en esta prueba fueron recogidos en pupas de Iridopsis sp. (Lepidoptera: Geometridae) en Curvelo, Minas Gerais, Brasil.

Hiljie y Cornelius (2001) reportan que Hypsipyla grandella puede ser considerada como la principal plaga forestal de América Latina y el Caribe, pues apenas una lagarta por árbol resulta en un daño severo, por presentar especificidad por los miembros de la subfamilia Swietenoideae de las Meliáceas, con amplia distribución geográfica, además de atacar varias estructuras del árbol (hojas, fuste y frutos). Se observa que el mayor daño consiste en la perforación de los brotes nuevos, resultando en la ramificación del fuste. Este hecho implica consecuencias económicas graves, ya que el período crítico para el ataque se da en los tres primeros años del cultivo del árbol, afectando la troza basal que es la más valiosa, por lo cual la bifurcación en una planta nueva no produciría madera de valor comercial, el ataque además retardaría el crecimiento, acarreando un aumento de los costos de manutención, de por sí altos principalmente en los primeros años de plantación.

Las evidencias muestran que las lagartas pasan muy poco tiempo fuera del brote, básicamente algunos segundos al nacer, antes de perforar la planta, motivo por el cual el control químico no resulta efectivo, vinculado también a la alta pluviosidad de las localidades donde las meliáceas son plantadas (Grijpma, 1974; Newton et al., 1993; Ohashi et al., 2002).

En el área experimental del Laboratorio de Control Biológico de Plagas Florestales del Departamento de Producción Vegetal, de la Faculdad de Ciencias Agronómicas, Universidad Estadual Paulista Julio Mesquita Filho - Campus de Botucatu, São Paulo, Brasil, esta especie ha sido criada bajo condiciones controladas sobre plantas de Swietenia macrophylla. Las pupas de $H$. grandella obtenidas de esa cría fueron acondicionadas en potes plásticos, mantenidas a temperatura de $26 \pm 2{ }^{\circ} \mathrm{C}$, humedad relativa de $60 \pm 10 \%$ y fotofase de 12 horas. Después de 17 días, especímenes del parasitoide emergieron de una de esas pupas, los que fueron mantenidos en vidrios (2,2 cm de diámetro $\mathrm{x} 14,0 \mathrm{~cm}$ de altura), identificados, cubiertos con tejido de tipo organza, 
conteniendo en su interior una gota de miel pura para la alimentación de los adultos, además de colocar nuevas pupas de $H$. grandella de 24 a 72 horas de edad para la multiplicación. Especímenes de este parasitoide fueron removidos de los tubos después de 24 horas, preservados en alcohol al 70\% y enviados al Instituto biológico, en Campinas, São Paulo, Brasil, para su identificación.
El parasitoide fue identificado como Trichospilus diatraeae Cherian \& Margabandhu, 1942 (Hymenoptera: Eulophidae). Esto representa el primer reporte de este parasitoide en una nueva especie $H$. grandella (Lepidoptera: Pyralidae) en Brasil. Esto abre perspectivas para su investigación tendientes a determinar el potencial de $T$. diatraeae para el control biológico de $H$. grandella.

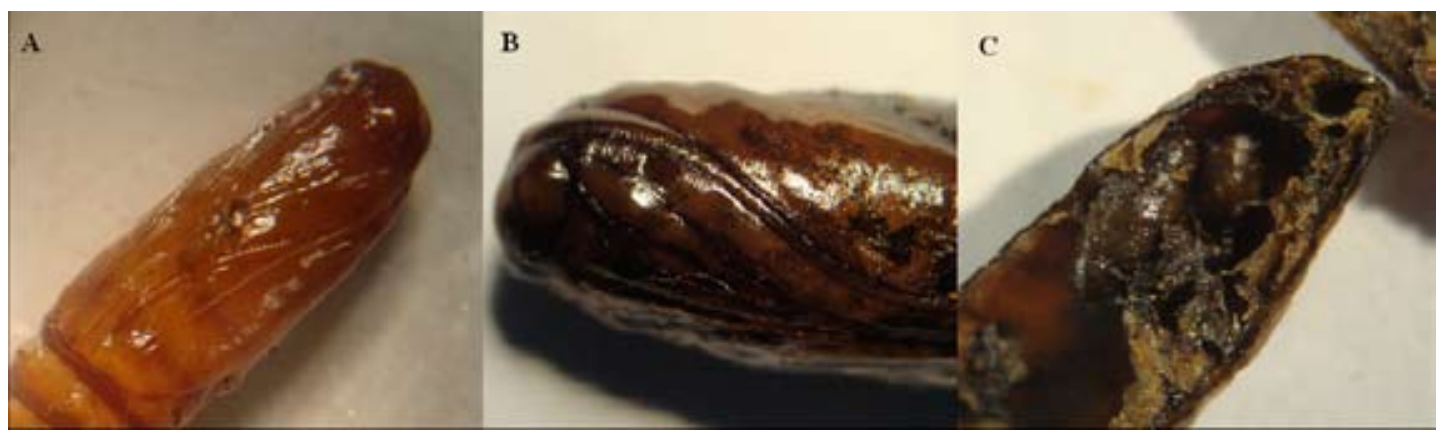

Figura 1. Hembra de Trichospilus diatraeae parasitando H. grandella (A); orificio de emergencia de los parasitoides (B); pupas de los parasitoides dentro de pupas de $H$. grandella (C).

\section{LITERATURA CITADA}

BENNETT, F.D.; GLENN, H.; YASEEN, M.; BARANOWSKI, R.M. 1987. Records of Trichospilus diatraeae, an Asian parasite (Hymenoptera: Eulophidae) from the Caribbean and Florida. Florida Entomologist, 70: 184-186.

BETBEDER-MATIBET, M. 1990. Élevage de plusieurs espéces du genre Chilo et de certains de leurs parasites pour la lutte biologique contre les foreurs des graminees en Afrique. Insect Science and Application, 11: 617-623.

BORDAT, D.; BRENIERE, J.; COQUARD, J. 1977. Foreurs de gramines africaines: parasitisme et techniques d'élevage. Agronomie Tropicale, 32: 391-399.

BOUCEK, Z. 1976. The African and Asiatic species of Trichospilus and Cotterellia (Hymenoptera: Eulophidae). Bulletin of Entomological Research, 65: 669-681.

BOURNIER, J.P. 1975. Sur la reproduction parthenogenetique de Trichosplilus diatraeae Cher. et Margab. (Hymenoptera: Chalcidoidea). Bulletin de la Societé Entomologique de France, 80: 116-118.

BRENIÈRE, J.; BORDAT, D.; VERCAMBRE, B.; HAMZA, H.; RENAND, M. 1985. Les opérations de lutte biologique contre lê foreur du maïs Chilo partellus (Swinhoe), Lepidoptera, dans L'île de Ngazidja. Agronomie Tropicale, 40: 157-166.

CHERIAN, M.C.; MARGABANDHU, V. 1942. A new species of Trichospilus (Hymenoptera: Chalcidoidea) from South India. Indian Journal of Entomology, 4: 101-102.

ÈTIENNE, J. 1975. Notes sur l'élevage et la biologie de Pseudoperichaeta laevis (Diptera: Tachinidae) sur hote de laboratoire. Entomophaga, 20: 105-111.

ÈTIENNE, J.; VIETTE, P. 1973. Nouvelle note sur Polydesma umbricola Boisduval (Lepidoptera: Noctuidae). Bulletin de la Société Entomologique de France, 78: 98-107.
FERRIERE, C. 1930. Notes on Asiatic Chalcidoidea. Bulletin of Entomological Research, 21: 353-360.

GRIJPMA, P. 1971. Studies on the shootborer Hypsipyla grandella Zeller observations on a rearing technique and on host seletion behavior of adults in captivity. Revista Interamericana de Ciências Agrícolas, 21 (2): 202-213.

HILJIE, L.; CORNELIUS, J. 2001. Es inmanejable Hypsipyla grandella como plaga florestal. Turrialba. Hoja tecnica del CATIE (18): 1-4.

NEWTON, A. C.; BAKER, P.; RAMNARINE, S.; MESÉN, J. F.; LEAKEY, R. R. B. 1993. The mahogany shoot-borer: prospects for control. Forest Ecology and Management, 57: 301-328.

NOYES, J. 2003. Universal Chalcidoidea Database. Disponivel em: http://www.nhm.ac.uk/research-curation/projects/ chalcidoids. Acesso em: 20 out. 2009.

OHASHI, O. S. 2002. Manejo Integrado da Broca do Mogno - Hypsipyla grandella Zeller (Lep. Pyralidae). In: Manejo Integrado das Principais Pragas e Doenças de Cultivos Amazônicos, Belém-Pa, pp. 91-120.

OLIVEIRA, H.N.; ZANUNCIO, J.C.; PEDRUZZI, E.P.; ESPFNDULA, M.C. 2005. Rearing of Thyrinteina arnobia (Lepidoptera: Geometridae) on guava and eucalyptus in laboratory. Brazilian Archives of Biology and Technology. 48: 801-806.

PARON, MR.; BERTI-FILHO, E. 2000. Capacidade reprodutiva de Trichospilus diatraeae (Hymenoptera: Eulophidae) em pupas de diferentes hospedeiros (Lepidoptera). Scientia Agricola. 57: 355-358.

PEREIRA, F.F.; ZANUNCIO, J.C.; TAVARES, M.T.; PASTORI, P.L.; JACQUES, G.C. 2008. Record of 
Trichospilus diatraeae (Hymenoptera: Eulophidae) as parasitoid of the eucalypt defoliator Thyrinteina arnobia (Lepidoptera: Geometridae) in Brazil. Phytoparasitica, 36: 304-306.

RODRIGUEZ-DEL-BOSQUE, L.A.; SMITH JUNIOR, J.W. 1989. Parasitization of Diatraea lineolata pupa and diapausing larvae by several exotic parasites. Florida Entomologist, 72: 703-705.

TORRES-BAUZA, J.A. 1994. Hymenopterous parasitoids of Dismorfia spio (Pieridae: Dismorphiinae). Journal of the Lepidopterist's Society, 48: 266.
UBAIDILLAH, R. 2006. Eulophine parasitoids of the genus Trichospilus in Indonesia, with the description of two new species (Hymenoptera: Eulophidae). Entomological Science, 9: 217-222.

ZACHÉ, B.; WILCKEN, C.F.; DACOSTA, R.R.; SOLIMAN, E.P. 2010. Trichospilus diatraeae Cherian \& Margabandhu, 1942 (Hymenoptera: Eulophidae), a new parasitoid of Melanolophia consimilaria (Lepidoptera: Geometridae). Phytoparasitica, 38: 355-357.

ZHU, C.D.; LASALLE, J.; HUANG, D.W. 2002. A study of Chinese Cirrospilus Westwood (Hymenoptera: Eulophidae). Zoological Stududies, 41: 23-46. 